\title{
FORTALECIMIENTO DE LOS SISTEMAS AGRÍCOLAS PRODUCTIVOS EN LA ZONA DE AMORTIGUAMIENTO DEL PARQUE NACIONAL VIÑALES: una alternativa hacia el desarrollo sostenible de las comunidades rurales
}

\author{
STRENGTHENING AGRICULTURAL PRODUCTION SYSTEMS IN \\ THE BUFFER ZONE OF THE PROTECTED AREA NATIONAL PARK \\ VIÑALES: an alternative to sustainable development of rural communities
}

\author{
Yoel Martínez Maqueira \\ Centro de Investigaciones y Servicios Ambientales. ECOVIDA. Pinar del Río. Cuba \\ yoel@ecovida.cu \\ Ignacio H. Diaz-Marroto \\ Departamento Ingeniería Agroforestal. Escuela Politécnica Superior. Universidad Santiago de \\ Compostela. España \\ Escuela Politécnica Superior. Departamento Ingeniería Agroforestal. Universidad Santiago de \\ Compostela. 27002. Lugo. España \\ ignacio.diazmaroto@usc.es \\ Ernesto Mujíca Benitez \\ Jardín Botanico Soroa. Universidad Artemisa \\ emujica@upr.edu.cu \\ José Luis Corvea Porras \\ Inversiones Gamma.S:A \\ joseluis.corvea@gmail.com \\ Ana M. Castro Barrio \\ Centro de Investigaciones y Servicios Ambientales. ECOVIDA. Pinar del Río. Cuba \\ germy@ecovida.cu
}

\section{Resumo}

A perda e degradação do habitat, como resultado de práticas agrícolas insustentáveis, poluição e a presença de espécies exóticas, continuam sendo as ameaças predominantes para a diversidade biológica terrestre e aquática. Todas contribuem para um declínio nos serviços ecossistêmicos, que pode envolver aumento da insegurança alimentar e colocar em risco a redução da pobreza e a melhoria da saúde e do bem-estar humano. Devido à agricultura acima indicada estar reorientou apoiada por técnicas ecológicas nas comunidades associadas à ANP Parque Nacional de Viñales, proporcionando maior desempenho, produtividade e eficiência econômica, sob forma sustentável e ambientalmente compatível à óptica ambiental, contribuindo para a melhoria da qualidade de vida da população local. Nove unidades de pesquisa agrícola localizados em comunidades rurais de El Capón, El Palmarito, El Cuajaní, La Ceiba y Pan de Azúcar foram selecionados. Nestas, foi realizada uma avaliação do comportamento dos indicadores de sustentabilidade em nível fazenda através da análise de sistemas agrícolas na perspectiva econômica, social e ecológica. No estudo sobre a avaliação da participação e integração das comunidades locais no Parque Nacional Viñales (Cuba), as alternativas de produção propostas relacionadas com a agricultura sustentável se desenvolve. Os resultados obtidos são baseados na participação das comunidades no uso de boas práticas, 
Fortalecimiento de los sistemas agrícolas productivos en la zona de amortiguamiento del Parque Nacional Viñales: una alternativa hacia el desarrollo sostenible de las comunidades rurales
Yoel Martínez Maqueira

Ignacio H. Diaz-Marroto

Ernesto Mujíca Benitez

José Luis Corvea Porras Ana M. Castro Barrio

fortalecimento dos sistemas agrícolas. Antes da colocação em funcionamento de tais práticas, as comunidades não tinham um plano de gestão integrada de áreas protegidas no Sistema Nacional de Áreas Protegidas (SNAP). Para a gestão participativa, que contribuíram para a identificação e incorporação de novos indicadores na eficácia da gestão, através de uma abordagem participativa das comunidades que afetam a gestão da área protegida; caracterizar os sistemas produtivos agrícolas e avaliar a sustentabilidade alcançada em fazendas selecionadas.

Palavras-chave: Desenvolvimento rural. Comunidades rurais. Sustentabilidade participativa. Agroecologia. Gestão participativa. ANP Parque Nacional Viñales (Cuba).

\section{Summary}

The loss and degradation of habitat as a result of unsustainable farming practices, pollution and the presence of exotic species, remain the predominant threats to terrestrial and aquatic biological diversity. All of them contribute to a decline in ecosystem services, which may involve increasing food insecurity and endanger reducing poverty and improving health and human welfare. Due to the above stated reoriented farming supported by ecological techniques in communities associated with ANP Viñales National Park, providing increased performance, productivity and economic efficiency, under a sustainable and environmentally compatible optical environment, contributing to improving the quality of life of local people. In nine agricultural research farms located in rural communities in El Capon, The Palmarito The Cuajaní, La Ceiba y Pan de Azúcar were selected. In these, an assessment of the behavior of the sustainability indicators at the farm level was performed by analyzing agricultural systems from an economic, social and ecological perspective. In the research study on the evaluation of the participation and integration of local communities in the National Park Viñales $(\mathrm{Cuba})$ in the proposed production alternatives related to sustainable agriculture develops. The results obtained are based on the participation of communities in the use of good practices, strengthening agricultural systems. Prior to the commissioning of these practices, communities lacked an integrated management plans of protected areas in the National System of Protected Areas (SNAP) .For participative management which it contributed to the identification and incorporation of new indicators in management effectiveness, through a participatory approach of communities that affect the management of the protected area; further characterize the productive agricultural systems and evaluate the sustainability achieved on farms selected.

Keywords: Rural development. Rural communities. Participatory sustainability. Agroecology. Participatory management. ANP National Park Viñales (Cuba).

\section{Introducción}

En el mundo actual, existe cierta tendencia, a que cada país alcance su soberanía alimentaria basándose en sistemas de producción agroecológicos, mediante la aplicación de la Agroecología Moderna, estos sistemas de conocimientos tradicionales, aportados por las 
Fortalecimiento de los sistemas agrícolas productivos en la zona de amortiguamiento del Parque Nacional Viñales: una alternativa hacia el desarrollo sostenible de las comunidades rurales
Yoel Martínez Maqueira

Ignacio H. Diaz-Marroto

Ernesto Mujíca Benitez

José Luis Corvea Porras Ana M. Castro Barrio

comunidades campesinas y académicas, han demostrado que se puede mejorar la seguridad alimentaria, al mismo tiempo, que se conservan los recursos naturales, la agro-biodiversidad y la conservación del agua y el suelo en las comunidades rurales (PRETTY et al., 2003).

Numerosos estudios coinciden en que la escasez de alimentos no es un problema en el mundo de hoy. En realidad, la cuestión radica en la forma en que los alimentos son producidos, distribuidos y utilizados. Tampoco es cierto que nuestro planeta carezca de suficientes recursos naturales; existe suficiente tierra, agua y diversidad genética para cubrir las demandas de la población mundial. El problema está en la velocidad con que estos recursos son depredados debido a prácticas ambientales inadecuadas.

En la actualidad, existen diversos criterios entre investigadores, especialistas, ecólogos, agroecólogos y conservacionistas sobre la compatibilidad de la agricultura con la conservación de los recursos naturales y, especialmente, en las áreas naturales protegidas. Desde la antigüedad, el hombre hizo uso de los recursos naturales para su existencia, como parte del ecosistema, y dependiendo de ello para el consumo de alimentos y otros productos. En la búsqueda por reinstalar una racionalidad más ecológica en la producción agrícola, los científicos han ignorado un aspecto esencial o central en el desarrollo de una agricultura más autosuficiente y sostenible, "el entendimiento más profundo de la naturaleza de los agroecosistemas y de los principios por los cuales estos funcionan" (ALTIERI y TOLEDO, 2011).

Las necesidades y deseos de la creciente población humana han incrementado la extracción de los recursos bióticos y abióticos hasta el punto de extinguir y perturbar gravemente especies, poblaciones y ecosistemas con consecuencias devastadoras para la vida del planeta, lo que ha generado la necesidad de cambiar los hábitos de consumo y realizar un aprovechamiento de los recursos de manera racional y sostenible que garanticen la vida en la Tierra.

La mayoría de las comunidades rurales y las familias agricultoras tradicionales, a pesar, de las fluctuaciones climáticas, son capaces de enfrentarse a estos cambios (ALTIERI y KOOHAFKAN, 2008). De hecho, muchos agricultores se adaptan y hasta se preparan para el cambio climático, minimizando el fracaso de las cosechas por medio de un mayor uso de variedades locales tolerantes a la sequía, sistemas de cosecha de agua, policultivos, manejo

CAMPO-TERRITÓRIO: revista de geografia agrária, v. 11, n. 24, p. 06-26, abr., 2017 
Fortalecimiento de los sistemas agrícolas productivos en la zona de amortiguamiento del Parque Nacional Viñales: una alternativa hacia el desarrollo sostenible de las comunidades rurales
Yoel Martínez Maqueira

Ignacio H. Diaz-Marroto

Ernesto Mujíca Benitez

José Luis Corvea Porras Ana M. Castro Barrio

orgánico del suelo, recolección de plantas silvestres, agrosilvicultura y otras técnicas agrícolas tradicionales.

Existe un consenso entre los diferentes actores sociales e instituciones en la búsqueda con urgencia de alternativas de desarrollo rural desde la perspectiva de la sostenibilidad, siendo la agroecología la vía más eficiente y viable para lograr la equidad en el mundo actual. GUZMAN et al. (2000), comentan que esta ciencia surgió como respuesta a la crisis ecológica en el campo agrícola, promocionando el manejo sostenible de los recursos naturales y el acceso igualitario a los mismos.

A pesar de esta evolución, el sector agrícola campesino o las pequeñas fincas agrícolas que abarcan disímiles estilos de agricultura con base ecológica, ofrecen modelos prometedores para la promoción de la diversidad biológica y para lograr rendimientos adecuados sin la utilización de agroquímicos, logrando además la conservación de la integridad ecológica de los agroecosistemas (HOLT GIMENEZ y PATEL, (2009). Según GLIESSMAN (1998), la agroecología enfatiza la capacidad de las comunidades locales para innovar, evaluar y adaptarse a condiciones heterogéneas extremas, a través de métodos de investigación participativa y de la extensión campesino a campesino, posteriormente SEVILLA-GUZMÁN et al., (2006) expresaron que las comunidades han de ser los protagonistas de un desarrollo rural participativo que encare la actual crisis medioambiental y sociocultural.

Los sistemas agroecológicos están profundamente arraigados en la racionalidad ecológica de la agricultura tradicional (TOLEDO, 1990; ALTIERI, 2004). Existen muchos ejemplos de sistemas agrícolas exitosos, caracterizados por su gran diversidad de cultivos y de animales domesticados, por el mantenimiento y mejora de las condiciones edáficas y por la gestión del agua y su biodiversidad, basados todos ellos en conocimientos tradicionales (TOLEDO y BARRERA-BASSOLS, 2008). Estos sistemas agrícolas no sólo han alimentado a gran parte de la población mundial, en diferentes partes del planeta, particularmente en los países en desarrollo, sino que también ofertan muchas de las posibles respuestas a los retos de la producción y la conservación de los recursos naturales que afectan al medio rural (KOOHAFKAN y ALTIERI, 2011).

Un aspecto importante a tener en cuenta en la actualidad y que tiene relación con la agricultura y la conservación de los recursos naturales, es la relación entre el cambio climático 
Fortalecimiento de los sistemas agrícolas productivos en la zona de amortiguamiento del Parque Nacional Viñales: una alternativa hacia el desarrollo sostenible de las comunidades rurales
Yoel Martínez Maqueira

Ignacio H. Diaz-Marroto

Ernesto Mujíca Benitez

José Luis Corvea Porras Ana M. Castro Barrio

y la agricultura, ya que, los campesinos están respondiendo al cambio climático minimizando la pérdida de cosechas a través de un mayor uso de variedades locales tolerantes a la sequía, aprovechando el agua, sembrando cultivos mixtos, creando sistemas agrosilvícolas, realizando prácticas de conservación de suelos e implementando otras técnicas tradicionales (ALTIERI y KOOHAFKAN, 2008). El análisis sobre el comportamiento de la agricultura después de fuertes eventos climáticos, ha puesto de manifiesto que la resistencia a los desastres climáticos está estrechamente relacionada con la biodiversidad presente en los sistemas productivos. Una encuesta realizada en América Central después del paso del huracán Mitch (HOLT-GIMENEZ, 2001) mostró que los campesinos que utilizan prácticas de diversificación, como cultivos de cobertura, cultivos intercalados y agrosilvicultura, sufrieron menos daños que sus vecinos con monocultivos convencionales.

Recientemente, estudios realizados por diferentes instituciones científicas, realizaron diversas encuestas en fincas campesinas en las provincias de Holguín y Las Tunas, después que de que el huracán Ike azotará a Cuba en 2008, y se encontró que las fincas diversificadas mostraron pérdidas de un 50\%, en comparación con un 90 y hasta el $100 \%$ en monocultivos vecinos. Asimismo, fincas agroecológicas mostraron una recuperación productiva más rápida (80 a 90\%, 40 días después del huracán), que las fincas de monocultivo (MACHÍN et al., 2010; ROSSET et al., 2011). Los estudios antes mencionados enfatizan la importancia de mejorar la diversidad vegetal y la complejidad en los sistemas agrícolas para reducir la vulnerabilidad a eventos climáticos extremos.

Evaluaciones realizadas en Asia, África y América Latina, muestran que las tecnologías agroecológicas pueden resultar beneficiosas para los agricultores y las comunidades, tanto en términos ambientales como económicos (GLIESSMAN, 2002).

Algunos gobiernos, pero sobre todo muchas organizaciones no gubernamentales y movimientos sociales están desarrollando este enfoque agroecológico (CAPORAL y MORALES, 2004) para dar respuesta a una producción agraria desarticulada $\mathrm{y}$, a veces, insuficiente. Actualmente se reconoce que la relación entre la conservación de la diversidad biológica y las necesidades de desarrollo de las comunidades locales es un elemento clave en la administración de los espacios protegidos, siendo estos espacios clave para promover investigaciones multidisciplinarias en beneficio de la conservación y el uso sostenible de los recursos naturales.

CAMPO-TERRITÓRIO: revista de geografia agrária, v. 11, n. 24, p. 06-26, abr., 2017 
Fortalecimiento de los sistemas agrícolas productivos en la zona de amortiguamiento del Parque Nacional Viñales: una alternativa hacia el desarrollo sostenible de las comunidades rurales
Yoel Martínez Maqueira

Ignacio H. Diaz-Marroto

Ernesto Mujíca Benitez

José Luis Corvea Porras Ana M. Castro Barrio

En la siguiente investigación se realizó una evaluación de los indicadores de sostenibilidad identificados a nivel de finca, evaluándose las condiciones de estas, antes y después de las acciones de manejo emprendidas en el área de estudio.

Una vez obtenidos los resultados, se realizará una evaluación del nivel de sostenibilidad en nueve fincas, elaborándose doce indicadores para evaluar dicho nivel en cada una de ellas, obteniéndose una valoración cualitativa y cuantitativa de los indicadores de sostenibilidad identificados, evaluándose las condiciones de estas, antes y después de las acciones de manejo realizadas en el área de estudio.

Cada indicador identificado y evaluado permitirá conocer de manera particularizada las necesidades del manejo de cada sistema, mejorando la productividad, reducción de riesgos e incertidumbres, se aumentó los servicios ecológicos y socioeconómicos por parte de las familias campesinas, contribuyendo con ello a la protección y conservación de los recursos naturales, prevenir la degradación de los suelos, agua y biodiversidad, sin disminuir la viabilidad económica del sistema.

El estudio realizado tiene como objetivo caracterizar los sistemas agrícolas productivos en las zonas de amortiguamiento, mediante la evaluación de indicadores de sostenibilidad a nivel de finca, y analizar como incide su funcionamiento en el manejo y gestión de los recursos naturales presentes en el ANP Parque Nacional Viñales.

En la actualidad, con el desarrollo de la agroecología, como la alternativa más viable en un futuro inmediato, compiten muchos modelos en su desarrollo y aplicación, que tienen como fin lograr una agricultura eficiente, biodiversa, y resistente; aunque los objetivos de todos estos enfoques pueden ser similares, sin embargo, cuando se examinan los atributos básicos de un sistema de producción sostenible, los enfoques agroecológicos cumplen con la mayoría de estos atributos y requisitos (ALTIERI, 2002; GLIESSMAN, 1998; UPHOFF, 2002; UK FOOD GROUP, 2010). Recientemente un estudio realizado por KOOHAFKAN y AlTIERI (2011), diseñó un grupo de preguntas orientadas a evaluar si los sistemas agrícolas propuestos contribuyen a los medios de vida rural sostenible

Actualmente, se desarrollan diferentes prácticas agroecológicas que están contribuyendo a medios de vida sostenibles mediante la mejora de los recursos naturales, humanos, físicos y el capital financiero de las comunidades rurales (KOOHAFKAN y ALTIERI, 2011). Son muchos los proyectos que se llevan a cabo en diferentes países en desarrollo con el objetivo 
Fortalecimiento de los sistemas agrícolas productivos en la zona de amortiguamiento del Parque Nacional Viñales: una alternativa hacia el desarrollo sostenible de las comunidades rurales
Yoel Martínez Maqueira

Ignacio H. Diaz-Marroto

Ernesto Mujíca Benitez

José Luis Corvea Porras Ana M. Castro Barrio

de elevar las producciones agrícolas mediante enfoques agroecológicos, alcanzando la seguridad alimentaria, el incremento de los ingresos y la resilencia al cambio climático, así como el empoderamiento de las comunidades rurales (PRETTY et al., 2003; REIJ y SMALIN, 2008; PRETTY et al., 2011).

\section{Materiales y métodos}

El estudio fue desarrollado en el Área Natural Protegida "Parque Nacional Viñales", localizada en la provincia Pinar del Río, municipio Viñales, Cuba, (Figura 1), insertándose al Sistema Nacional de Áreas Protegidas de Cuba, en el año 2001, en la categoría II de la IUCN, por encontrarse ubicado en la segunda región con un mayor número de endemismos del país, presenta una alta variedad de ecosistemas que permiten la existencia de diversos hábitats, debido a ello, existe una gran diversidad de flora y fauna. La flora está constituida por más de 1.200 especies, alcanzando un endemismo cercano al 30\%, en sustratos carbonatados, mientras la fauna presenta un endemismo muy elevado en grupos particulares, que como los moluscos, puede alcanzar un 90\%. Los anfibios, reptiles y crustáceos, presentan un porcentaje de endemismo similar a la flora (MARTINEZ, 2013).

Los excepcionales valores naturales de la provincia de Pinar del Río, la más occidental de la Isla de Cuba, históricamente han sido reconocidos, especialmente en el ámbito científico y académico. Por ello, a mediados del siglo pasado, se elaboraron propuestas de diferentes categorías de protección que incluían las principales alturas "cársicas" de la Sierra de los Órganos y una zona de Viñales. Estudios sobre la vegetación combinados con los elementos geomorfológicos (LEHMANN et al., 1956), despertaron un gran interés por los elementos bióticos existentes en el área. 
Fortalecimiento de los sistemas agrícolas productivos en la zona de amortiguamiento del Parque Nacional Viñales: una alternativa hacia el desarrollo sostenible de las comunidades rurales
Yoel Martínez Maqueira

Ignacio H. Diaz-Marroto

Ernesto Mujíca Benitez

José Luis Corvea Porras Ana M. Castro Barrio

Figura 1: Localización geográfica del área protegida Parque Nacional Viñales y su área de influencia socioeconómica

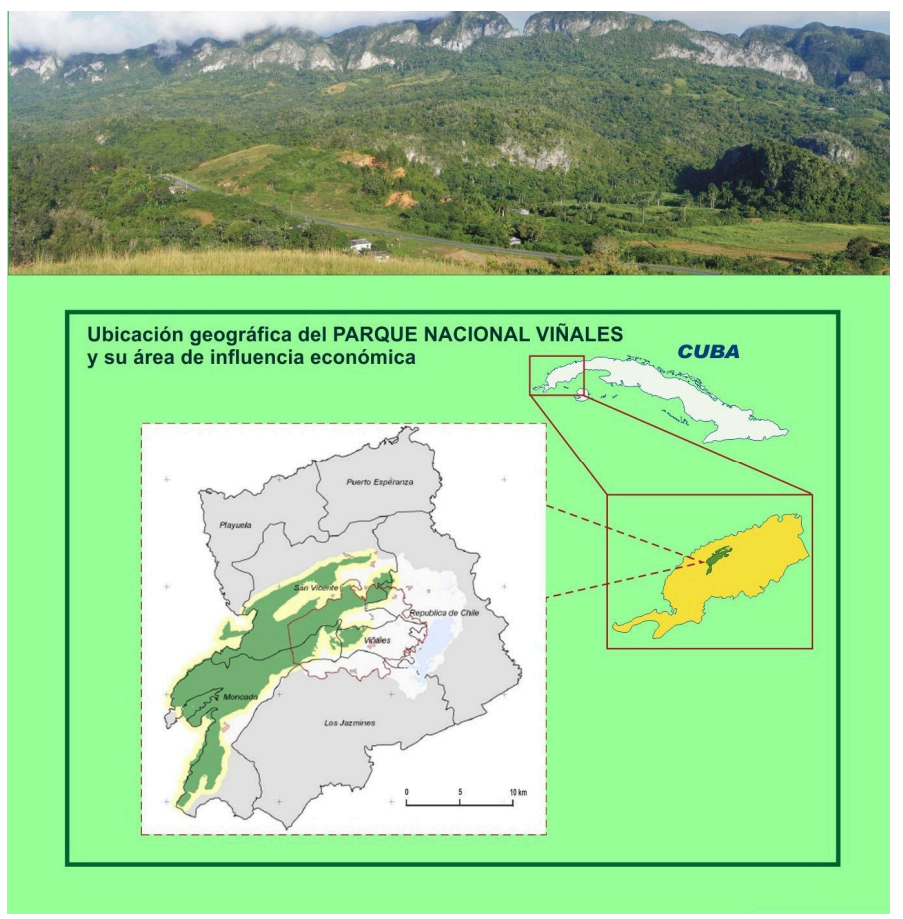

El Parque Nacional Viñales se encuentra ubicado en el occidente cubano, Distrito Pinareño del Sub-distrito Cordillera de Guaniguánico, porción centro-oriental de la región Sierra de los Órganos. Abarca las elevaciones de mogotes y pizarras, y algunos valles intramontanos que definen los tres tipos de paisajes con que cuenta esta ANP. Se han distinguido cuatro grupos de unidades de "nappes escamadas" (PSCZSOLKOWSKI, 1987): la Faja de Mogotes, las Alturas de Pizarras del Sur, las Alturas de Pizarras del Norte y las unidades metamórficas que ocupan la posición más elevada.

El mismo posee una superficie de 15010.0 ha, de ellas corresponden al área núcleo 11120 ha y 3820 ha a la zona de amortiguamiento, área donde se encuentran los sistemas agrícolas productivos, representados por un total 751 campesinos en las en las diferentes estructuras agrícolas existentes: Cooperativa de Créditos y Servicios (CCS), Cooperativa de Producción Agropecuaria (CPA) y Unidades Básicas de Producción Cooperativa (UBPC), aprovechándose estas para la siembra de los cultivos de arroz, maíz, frijol, hortalizas, tomate, tubérculos y raíces, cítricos, frutales, plátano y tabaco y otras producciones complementarias.

En el estudio y evaluación de los sistemas agroecológicos y su vinculación al manejo y gestión del espacio protegido, se seleccionaron nueve fincas agrícolas ubicadas en las

\section{CAMPO-TERRITÓRIO: revista de geografia agrária, v. 11, n. 24, p. 06-26, abr., 2017}


Fortalecimiento de los sistemas agrícolas productivos en la zona de amortiguamiento del Parque Nacional Viñales: una alternativa hacia el desarrollo sostenible de las comunidades rurales
Yoel Martínez Maqueira

Ignacio H. Diaz-Marroto

Ernesto Mujíca Benitez

José Luis Corvea Porras Ana M. Castro Barrio

comunidades rurales de: El Capón, El Palmarito, El Cuajaní, La Ceiba y Pan de Azúcar, esta última ubicada en el municipio Minas de Matahambre.

La selección de las fincas de estudio se realizó al azar, pero de manera sistemática en diferentes comunidades ubicadas en las zonas de amortiguamiento y de influencia socioeconómica, atendiendo a las especificidades de la actividad agrícola. Las muestras seleccionadas difieren cada una en sus características físicas, químicas, relieve, representatividad del paisaje, tradiciones locales propias,..., por lo que cada finca es una muestra particular para la investigación a desarrollar, (Tabla 1).

Los puntos de muestreo fueron seleccionados previamente como resultado de la implementación de algunos programas del plan de manejo, vinculados a la temática de estudio, estos se georreferenciaron con la utilización de un GPS con un margen de error inferior a $4 \mathrm{~m}$ (Figura 2).

Al inicio de la investigación, se realizó un diagnóstico individualizado, en cada finca, para comprobar que poseía características diferentes entre sus componentes abióticos y biológicos, así como en el uso del suelo y los cultivos. Además, las prácticas agroecológicas difieren en función de las experiencias y conocimientos empíricos adquiridos por cada campesino. El estudio se realizó en tres fases, durante los años 2009, 2011 y 2013, en cada una de las fincas seleccionadas.

Se inició con la visita a las fincas, para contactar, primeramente, con la Junta Coordinadora de cada Cooperativa de Créditos y Servicios (CCS), con el objetivo de explicar a los campesinos los detalles de la investigación a realizar y la necesidad de recopilar y obtener la información necesaria para el diagnóstico agroecológico. La información se centró en cuatro aspectos atendiendo a lo expresado por GLIESSMAN (1998), el cual plantea que el desarrollo agrícola involucra varios recursos adicionales al cultivo y afecta aspectos de la vida social, más allá del mero aumento de la producción.

También se realizó una revisión bibliográfica, así como la recopilación de información sobre temas relacionados con los indicadores de sostenibilidad a nivel de finca, así como con los diseños de fincas agroecológicas y las características biofísicas y socioeconómicas. 
Fortalecimiento de los sistemas agrícolas productivos en la zona de amortiguamiento del Parque Nacional Viñales: una alternativa hacia el desarrollo sostenible de las comunidades rurales
Yoel Martínez Maqueira Ignacio H. Diaz-Marroto Ernesto Mujíca Benitez José Luis Corvea Porras Ana M. Castro Barrio

Tabla 1: Localización de las fincas agrícolas

\begin{tabular}{|c|c|c|}
\hline Municipio & Nombre de la finca & Coordenadas \\
\hline \multirow{16}{*}{ Viñales } & \multirow{2}{*}{ El Paraíso } & N 22 $2^{\circ} 37^{\prime} 27,88^{\prime \prime}$ \\
\hline & & $\mathrm{W} 83^{\circ} 41^{\prime} 25,32^{\prime \prime}$ \\
\hline & \multirow{2}{*}{ La Ensenada } & $\mathrm{N} 22^{\circ} 38^{\prime} 21,01^{\prime \prime}$ \\
\hline & & W 83 $42^{\prime} 29,31^{\prime}$, \\
\hline & \multirow{2}{*}{ El Lele } & N $22^{\circ} 38^{\prime} 04,97^{\prime \prime}$ \\
\hline & & W $83^{\circ} 41^{\prime} 16,09^{\prime}$ \\
\hline & \multirow{2}{*}{ Los Esperones } & $\mathrm{N} 22 \cdot^{\circ} 38^{\prime} 41,35^{\prime}$, \\
\hline & & $\mathrm{W} 83^{\circ} 41^{\prime} 44,65^{\prime}$ \\
\hline & \multirow{2}{*}{ Los Nodas } & N $22^{\circ} 37^{\prime} 49,28^{\prime \prime}$ \\
\hline & & W $83^{\circ} 41^{\prime} 44,71^{\prime \prime}$ \\
\hline & \multirow{2}{*}{ Paco Hernández } & N $22 \cdot{ }^{\circ} 36^{\prime} 19,21$, \\
\hline & & W $83^{\circ}{ }^{\circ} 44^{\prime} 00,92^{\prime}$ ', \\
\hline & \multirow{2}{*}{ Félix Reyes } & N 22. ${ }^{\circ} 38^{\prime} 00,64^{\prime \prime}$ \\
\hline & & W $83^{\circ}{ }^{\circ} 41^{\prime} .49,21^{\prime}$, \\
\hline & \multirow{2}{*}{ Raúl Reyes } & $\mathrm{N} 22 \cdot{ }^{\circ} 37^{\prime} 18,22^{\prime}$, \\
\hline & & W $83^{\circ} 42^{\prime} 58,63^{\prime \prime}$ \\
\hline Mina de & \multirow{2}{*}{ Juanito Piara } & N 22. ${ }^{\circ} 36^{\prime} 51,21^{\prime \prime}$ \\
\hline Matahambre & & W 83.०49'38,91', \\
\hline
\end{tabular}

Figura 2: Localización de las fincas agriecológicas (zona de amortiguamiento y de influencia socioeconómica)

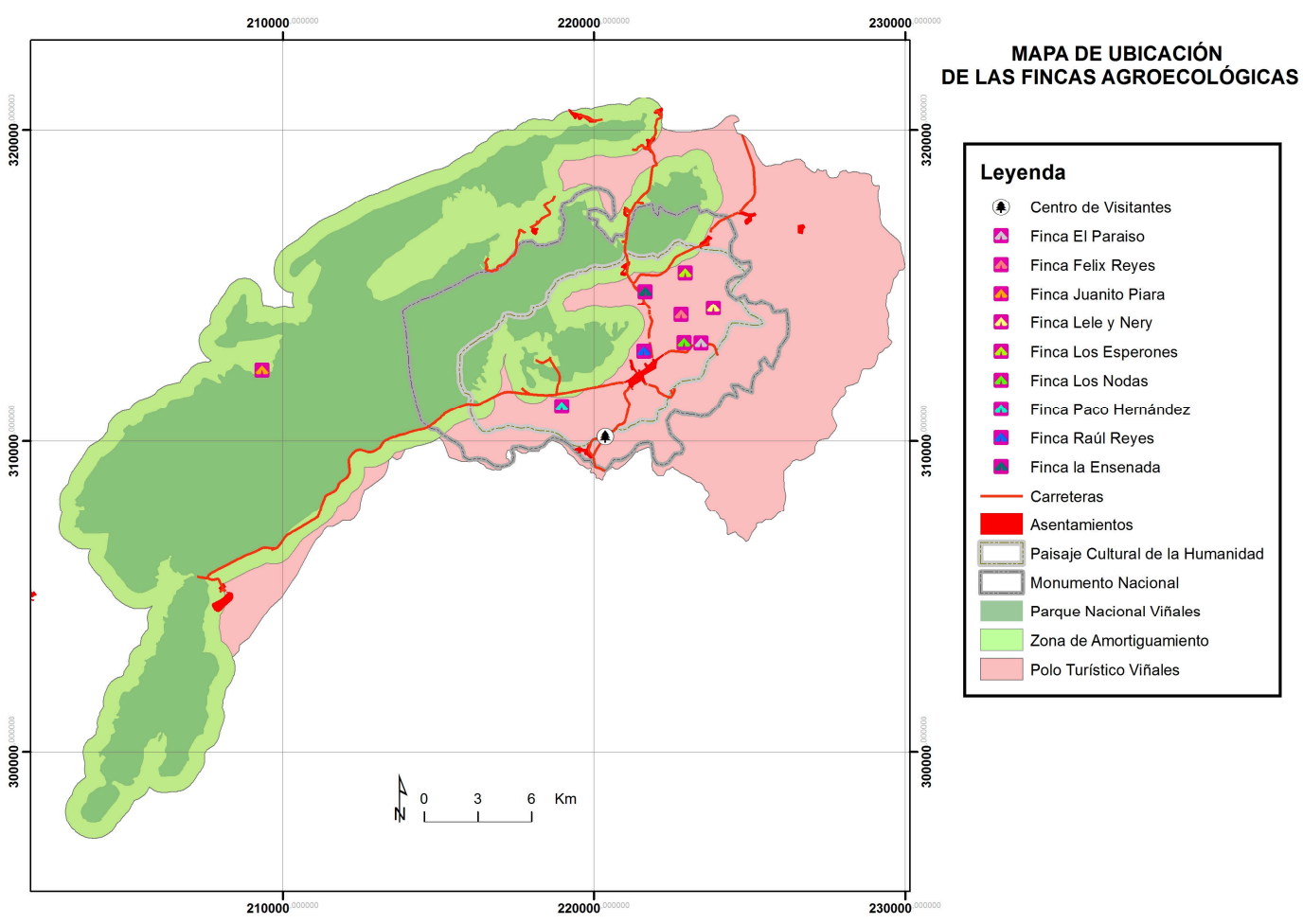


Fortalecimiento de los sistemas agrícolas productivos en la zona de amortiguamiento del Parque Nacional Viñales: una alternativa hacia el desarrollo sostenible de las comunidades rurales
Yoel Martínez Maqueira

Ignacio H. Diaz-Marroto

Ernesto Mujíca Benitez

José Luis Corvea Porras Ana M. Castro Barrio

\section{Indicadores de sustentabilidad}

Se utilizaron los mismos indicadores en cada finca, 12 en total, comparándose cada finca anualmente y, entre ellas, durante las 3 fases, siguiendo su trayectoria a través del tiempo y realizando comparaciones entre fincas en varios estados de transición. Para medir cada indicador, se diseñó una escala de 0 a 9. El rango menos óptimo es entre 0 y 3 , el medio entre 4 y 6 , y el óptimo entre 7 y 9. La evaluación de cada finca se realizó a través de criterios y diagnósticos que permiten construir indicadores del estado del sistema. Se inició el proceso de evaluación de sostenibilidad de acuerdo a los indicadores seleccionados, usando la metodología del “Índice de Valoración Ecológica de Agroecosistemas" (IVEA), con elementos MESMIS. (Tabla 2).

\section{Análisis estadistico}

Para el análisis estadístico se utilizó el paquete informático SPSS v19.0 y el software MATLAB 2011, para la confección de los gráficos. En el caso específico del análisis estadístico intragrupos (fincas), se utilizó un análisis estadístico no paramétrico (prueba $\mathrm{H}$ de Kruskal-Wallis), ya que los datos obtenidos no presentaban una distribución normal debido al escaso número de casos (indicadores) y para el análisis intergrupos se usó el test U de MannWhitney. Para la comparación entre fincas, anualmente, se realizó un análisis de varianza (ANOVA) de un factor, ya que, en este caso los datos mostraron una distribución normal. En el caso de la comparación interfincas se usó el método no paramétrico SNK (Studen-NewmKeuls).

Tabla 2: Indicadores a evaluar en cada área de estudio

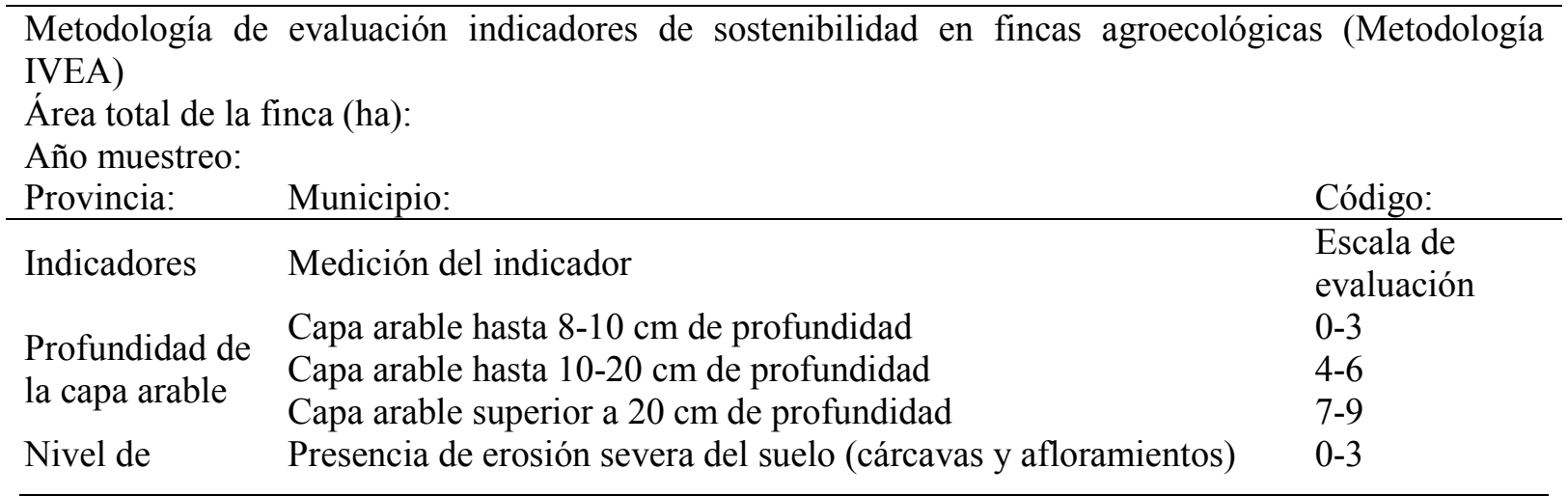

CAMPO-TERRITÓRIO: revista de geografia agrária, v. 11, n. 24, p. 06-26, abr., 2017 
Fortalecimiento de los sistemas agrícolas productivos en la zona de amortiguamiento del Parque Nacional Viñales: una alternativa hacia el desarrollo sostenible de las comunidades rurales
Yoel Martínez Maqueira

Ignacio H. Diaz-Marroto

Ernesto Mujíca Benitez

José Luis Corvea Porras Ana M. Castro Barrio erosión del

suelo

Nivel de compactación

del suelo

Diversidad

agrícola

Diversidad

forestal

Vida biológica

del suelo

Nivel de reciclaje del sistema

Conservación

de las

funciones

vitales del agroecosistema

Recursos de capital económico del agroecosistema

Manejo de plagas y enfermedades

Recursos de energía del agroecosistema

Recursos de capital social de la finca

Integración de los animales
Erosión intermedia sin daños significativos (zanjillas superficiales) 4-6

Baja intensidad de erosión con medidas de corrección 7-9

Lenta infiltración, con mal drenaje (muy compactado) 0-3

Moderada infiltración, con drenaje intermedio 4-6

Rápida infiltración, buen drenaje (suelo friable) 7-9

De 5 a 8 especies con 12 o 15 variedades de plantas comestibles 0-3

De 9 a 15 especies con 25 a 35 variedades de plantas comestibles 4-6

Más de 20 especies con 40 a 50 variedades de plantas comestibles 7-9

De 3-5 árboles/ha mayores de $3 \mathrm{~m} \quad 0-3$

De 9-11 árboles/ha mayores de $3 \mathrm{~m} \quad$ 4-6

De 12-15 árboles/ha mayores de $3 \mathrm{~m} \quad$ 7-9

Con poca o ninguna presencia de lombrices o artrópodos 0-3

Se observan hasta 30 lombrices o artrópodos por $\mathrm{m}^{2} \quad$ 4-6

Se observan más de 30 lombrices o artrópodos por $\mathrm{m}^{2} \quad$ 7-9

Sacar los residuos del campo y/o quemarlos $0-3$

Mezclar residuos con el suelo (inversión del prisma) 4-6

Residuos sobre la superficie y producción de abonos orgánicos 7-9

Sistemas no integrados, poca dependencia y baja calidad 0-3 paisajística

Aceptable integración en la finca, reducida presencia de hábitats naturales con problemas de conservación

Prácticas de policultivo, parches naturales de vegetación y buena calidad del paisaje

Capital insuficiente para el manejo de la finca 0-3

Capital suficiente para el acceso a medios de trabajo, semillas y conservación de suelos

Capital suficiente para el acceso a tecnologías, semillas, mejora

del suelo y cambios fundamentales en la vivienda

Uso mayoritario de productos químicos

$0-3$

Uso alternativo de productos químicos con rotaciones de cultivos 4-6

Uso mayoritario del manejo integrado de plagas y enfermedades 7-9

Alta dependencia de insumos externos

$0-3$

Combinación de insumos externos con tracción animal, energía humana y otros insumos de la finca

Uso suficiente de los insumos de la finca con la integración de plantas y animales y los procesos de reciclaje

Productor integrado a la comunidad con escasa capacitación y de innovación dentro de su finca

Productor y su familia realizan algunas actividades de innovación con actividades de capacitación

Productor y su familia integrados en proyectos de desarrollo comunitario. Alta capacidad innovadora en su finca y la 7-9 comunidad

Cantidad de animales mayores en la finca

Número de animales

\section{Resultados y discusión}

CAMPO-TERRITÓRIO: revista de geografia agrária, v. 11, n. 24, p. 06-26, abr., 2017 
Fortalecimiento de los sistemas agrícolas productivos en la zona de amortiguamiento del Parque Nacional Viñales: una alternativa hacia el desarrollo sostenible de las comunidades rurales
Yoel Martínez Maqueira

Ignacio H. Diaz-Marroto

Ernesto Mujíca Benitez

José Luis Corvea Porras Ana M. Castro Barrio

En la evaluación de cada finca se tuvieron en cuenta diversos aspectos para identificar qué tipo de buenas prácticas desarrollan los campesinos y como estas influyen en el nivel de sostenibilidad del agroecosistema.

El análisis estadístico realizado demuestra que existen diferencias significativas entre la media de las puntuaciones obtenidas en cada finca en el año 2009 (Figura 3), es decir, antes de la implementación de la metodología de la efectividad de manejo. No todas las fincas mostraron igual comportamiento y situación. La peor situación correspondió a las fincas, LEs (Los Esperones), EPa (El Paraíso) y PHz (Paco Hernández), mientras que las fincas RRe (Raúl Reyes) y LEn (La Ensenada), mostraron la situación más favorable

Figura 3: Comparación de las medias de las puntuaciones obtenidas en cada finca en el año 2009 (EPa: Finca El Paraíso, LEn: La Ensenada, ELe: Finca Lele y Nery, LEs: Finca los Esperones, LNo: Finca Los Nodas, FEx: Finca Félix Reyes, PHz: Finca Paco Hernández, JPa: Finca Juanito Piara, RRe: Finca Raúl Reyes)
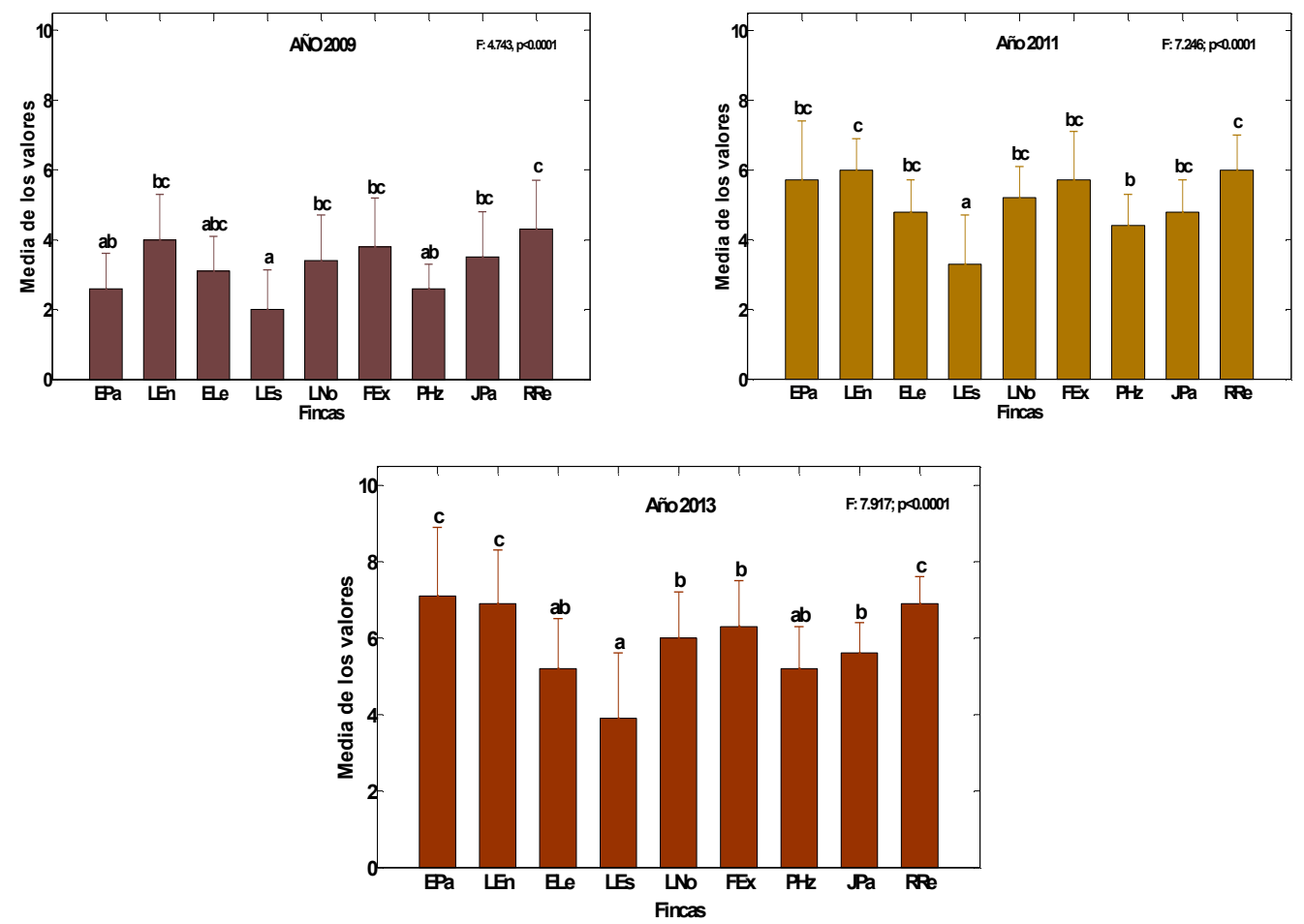

En la Tabla 3, se muestra el crecimiento de la evaluación de la efectividad de manejo de los indicadores de sostenibilidad con respecto a la media, al desarrollar las acciones de manejo y aplicar las técnicas agroecológicas, demostrándose que el agrosistema con una 
Fortalecimiento de los sistemas agrícolas productivos en la zona de amortiguamiento del Parque Nacional Viñales: una alternativa hacia el desarrollo sostenible de las comunidades rurales
Yoel Martínez Maqueira Ignacio H. Diaz-Marroto Ernesto Mujíca Benitez José Luis Corvea Porras Ana M. Castro Barrio

mayor efectividad corresponde a la finca El Paraíso (EPa), con un crecimiento de 4,5 veces el valor de la media.

Comparando todos los indicadores anualesentre las fincas y como se muestra en la Figura 3 El Paraiso (EPA) tiene una mayor eficacia en la implementación de los indicadores y gestión de los recursos naturales existentes, además incluyó todos los componentes de un sistema agroecológico, incluyendo el uso de la tierras agrícolas, planeamiento agrícola, eficiencia energetica del sistema, manejo inetgrado de plagas, actividades de formación y extensionismo y un alta vinculación de la familia en el procesos de producción.

Tabla 3: Crecimiento del valor medio de cada finca

\begin{tabular}{lc}
\hline Finca & $\begin{array}{c}\text { Crecimiento del } \\
\text { valor medio }\end{array}$ \\
\hline Finca El Paraíso (EPa) & 4.5 \\
Finca La Ensenada (LEn) & 2.9 \\
Finca Lele y Nery (ELe) & 2.1 \\
Finca Los Esperones (LEs) & 1.9 \\
Finca Los Nodas (LNo: & 2.6 \\
Finca Félix Reyes (FEx): & 2.5 \\
Finca Paco Hernández (PHz) & 2.6 \\
Finca Juanito Piara (JPa) & 2.1 \\
Finca Raúl Reyes (RRe) & 2.6 \\
\hline
\end{tabular}

Figura 4: Comparación de indicadores para cada finca agrícola en cada año evaluado

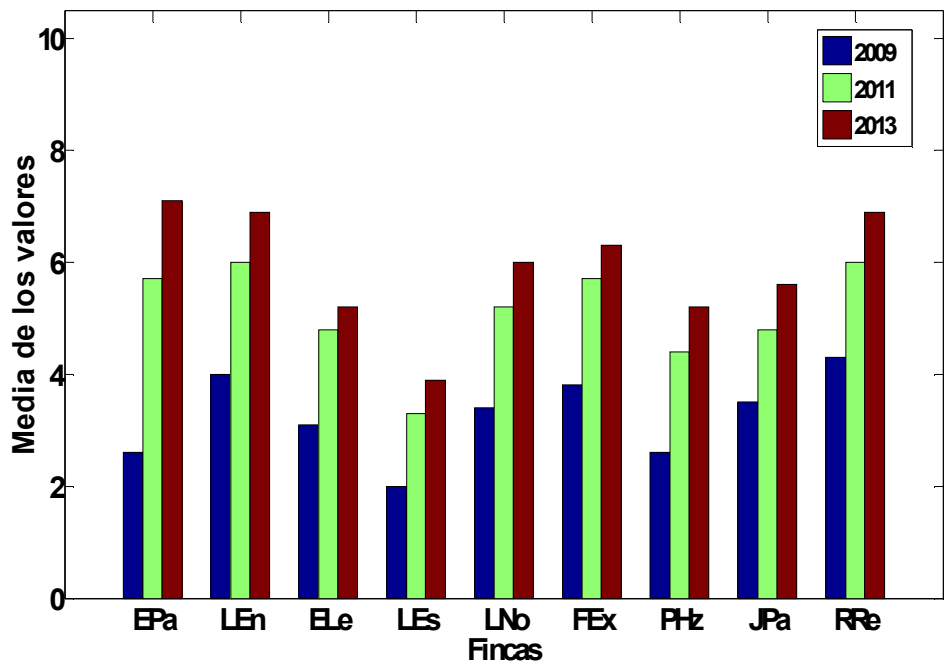

(EPa: Finca A Paraíso, LEn: O Enseada ELe: Finca Lele y Nery, LEs: Finca, LNo: A Finca Los Nodas, FEx: Finca Félix Reyes, PHz: Finca Paco Hernández, JPa: Finca Juanito Piara, RRe: Finca Raúl Reyes)Como se muestra en la Tabla 4, el comportamiento medio de los indicadores de sustentabilidad aplicado en las nueve fincas en los tres años de estudio. 
Fortalecimiento de los sistemas agrícolas productivos en la zona de amortiguamiento del Parque Nacional Viñales: una alternativa hacia el desarrollo sostenible de las comunidades rurales
Yoel Martínez Maqueira

Ignacio H. Diaz-Marroto

Ernesto Mujíca Benitez

José Luis Corvea Porras Ana M. Castro Barrio

Tabela 4: Valor médio de los indicadores de sustentabilidad alcanzada por cada finca

\begin{tabular}{|l|c|c|c|c|c|c|c|c|c|}
\hline \multicolumn{1}{|c|}{ Valor médio indicadores } & EPa & LEn & ELe & LEs & LNo & FEx & PHz & JPa & RRe \\
\hline $\begin{array}{l}\text { Profundidad de la capara } \\
\text { arable del suelo }\end{array}$ & 2.0 & 6.0 & 3.7 & 1.7 & 4.3 & 6.3 & 3.7 & 5.3 & 6.0 \\
\hline Nível de ersión del suelo & 4.7 & 6.3 & 57 & 2.3 & 5.3 & 6.3 & 3.7 & 4.3 & 6.7 \\
\hline Nível de compactación & 4.3 & 6.0 & 4.7 & 5.0 & 5.7 & 6.3 & 4.7 & 5.0 & 6.0 \\
\hline Diversidad agrícola & 6.3 & 7.0 & 2.7 & 2.3 & 6.3 & 5.0 & 3.0 & 4.7 & 6.0 \\
\hline Diversidad floristica & 6.3 & 4.3 & 4.3 & 5.3 & 5.3 & 6.3 & 2.7 & 4.0 & 6.0 \\
\hline Vida biologica del suelo & 5.0 & 4.3 & 4.0 & 2.3 & 4.3 & 4.0 & 4.0 & 3.7 & 5.0 \\
\hline Reciclaje a nivel de sistema & 3.7 & 4.7 & 3.7 & 2.7 & 33.3 & 4.0 & 3.7 & 4.0 & 5.3 \\
\hline $\begin{array}{l}\text { Conservación de las funciones } \\
\text { vitales del agroecosistema }\end{array}$ & 4.0 & 5.7 & 4.3 & 3.0 & 4.7 & 4.7 & 4.7 & 6.0 & 6.3 \\
\hline $\begin{array}{l}\text { Economía del agroecosistema } \\
\text { y recursos de capital. }\end{array}$ & 6.7 & 6.7 & 6.0 & 2.0 & 6.7 & 6.7 & 4.7 & 4.3 & 5.7 \\
\hline Pragas y enfermedades & 6.3 & 6.3 & 4.3 & 2.3 & 4.0 & 4.3 & 3.7 & 3.7 & 4.7 \\
\hline $\begin{array}{l}\text { Recursos de Energia } \\
\text { agroecossistema }\end{array}$ & 5.7 & 5.7 & 4.7 & 4.0 & 4.3 & 5.7 & 5.0 & 4.0 & 4.3 \\
\hline \begin{tabular}{l} 
Recursos de capital de la finca \\
\hline
\end{tabular} & 6.3 & 4.7 & 4.8 & 3.7 & 4.0 & 3.3 & 5.3 & 6.7 & 6.7 \\
\hline
\end{tabular}

La Figura 4, la sustentabilidad alcanzada y reflejada por las nueve fincas por los diferentes indicadores estudiados, ha existido una evolución cualitativa y cuantitativa de cada indicador, obteniendose un nivel de sustentabilidad agrícola. Es de resaltar que la finca con mayor dificultad en lograr un nivel de sustentabilidad óptimo es la finca de los Esperones (Les).

Posteriormente, como resultado de la integración y participación activa de las comunidades rurales en las zonas de amortiguación e influencia socioeconómica en las haciendas estudió la reforestación de áreas degradadas aumentó, reduciendo la presencia de especies exóticas invasoras. También identificar principales cuestiones ambientales asociadas con sistemas de producción agrícola y, al mismo tiempo, perjudicar la integridad de los ecosistemas de la ANP Parque Nacional de Viñales. 
Fortalecimiento de los sistemas agrícolas productivos en la zona de amortiguamiento del Parque Nacional Viñales: una alternativa hacia el desarrollo sostenible de las comunidades rurales
Yoel Martínez Maqueira Ignacio H. Diaz-Marroto Ernesto Mujíca Benitez José Luis Corvea Porras Ana M. Castro Barrio

Entre los indicadores de mayor efectividad y más cercano al óptimo se encuentran, el manejo de plagas y enfermedades, los recursos de capital económico del agroecosistema, los recursos de capital social de la finca y la diversidad agrícola y forestal, sin embargo, no ha ocurrido así, con los indicadores relacionados con la capa arable del suelo, por no ser este apto para el desarrollo de la mayoría de los cultivos, siendo este indicador uno de los puntos críticos del agro ecosistema.

A continuación relacionamos el comportamiento de los indicadores de sustentabilidad de cada sistema agrícola productivo mediante un gráfico de tipo Tela Araña, donde se muestra la evolución efectiva desde el año 2009-2013, identificándose los indicadores más cercanos al óptimo de sostenibilidad y los indicadores más críticos por cada finca estudiada.

Figura 5: Comportamineto de los indicadores por cada finca

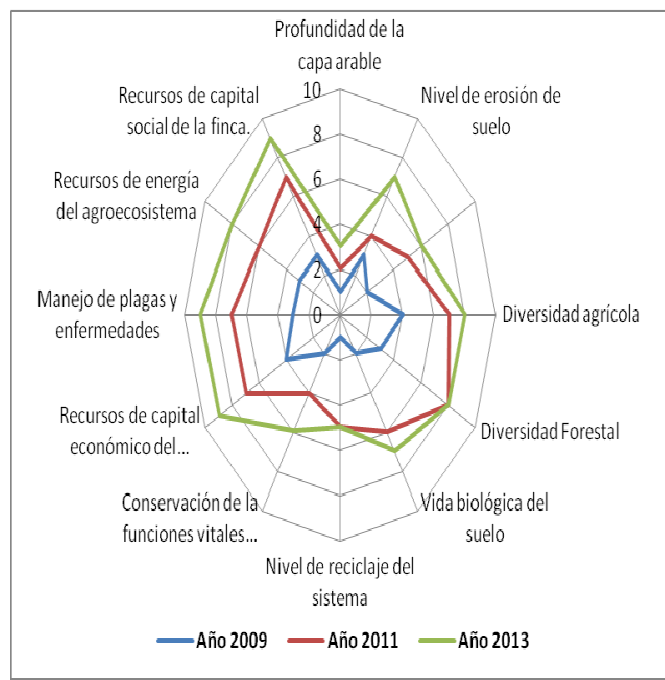

Finca El Paraíso

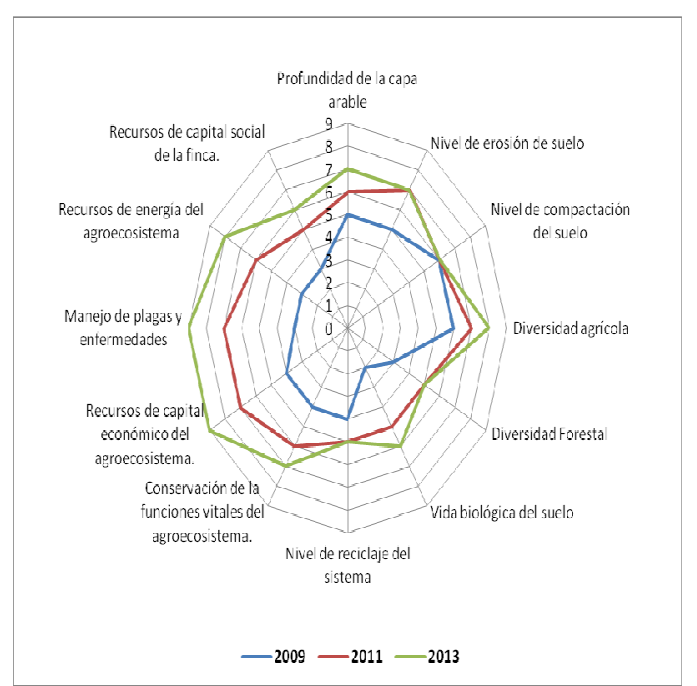

Finca la Ensenada 
Fortalecimiento de los sistemas agrícolas productivos

Yoel Martínez Maqueira en la zona de amortiguamiento del Parque Nacional

Ignacio H. Diaz-Marroto Viñales: una alternativa hacia el desarrollo

Ernesto Mujíca Benitez sostenible de las comunidades rurales

José Luis Corvea Porras Ana M. Castro Barrio

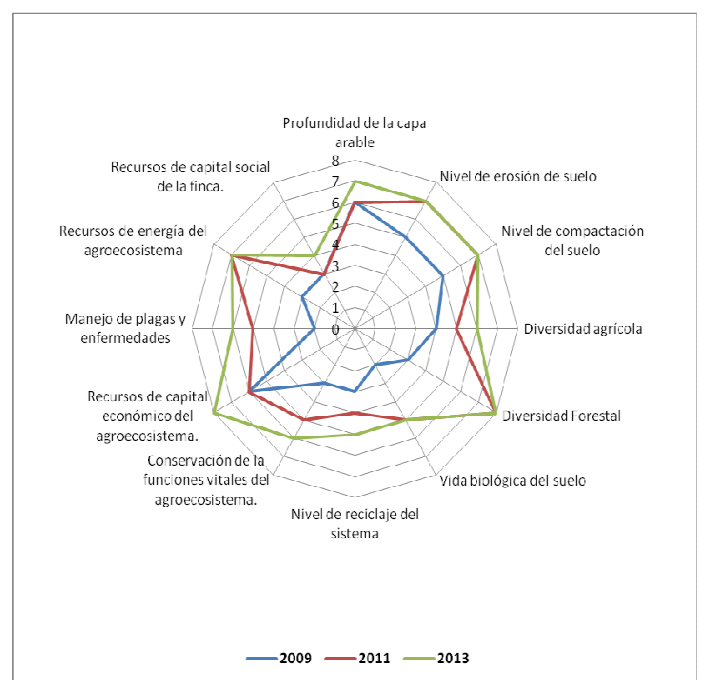

Finca Felix Reyes

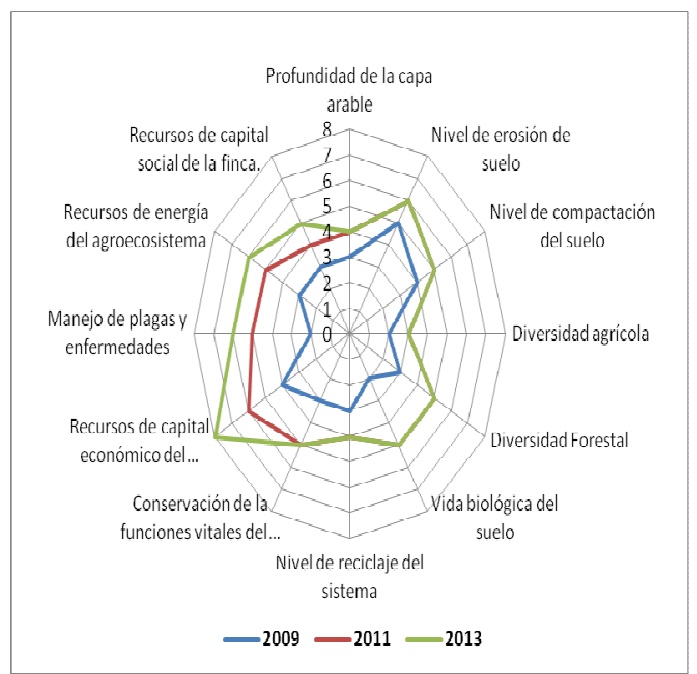

Finca Lele y Nery

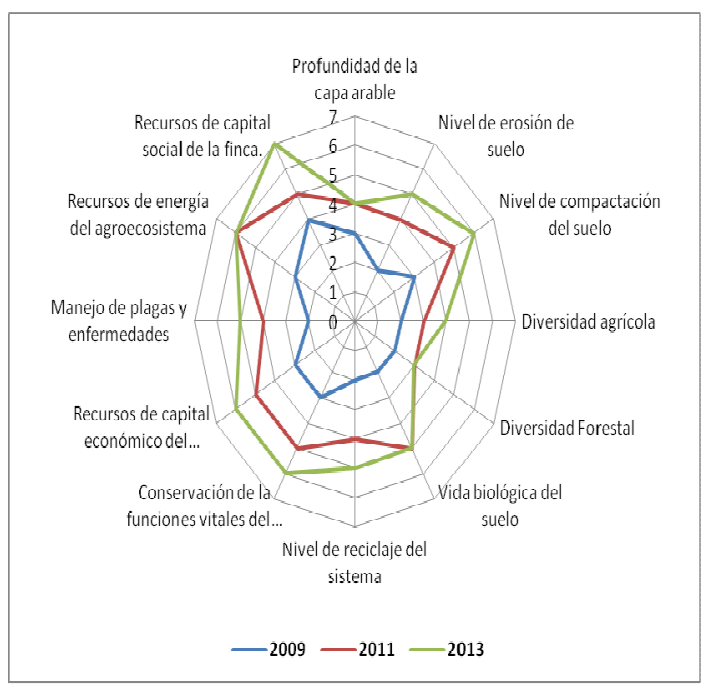

Finca Paco Hernández

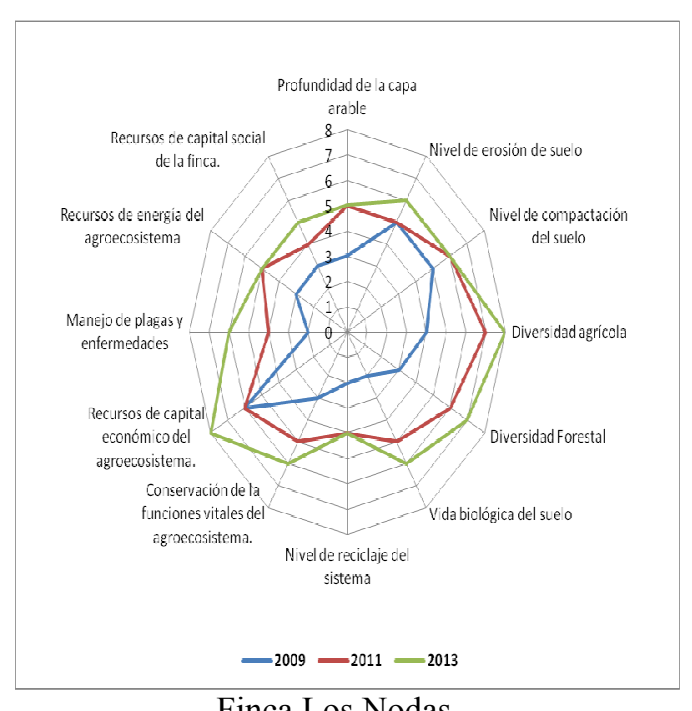

Finca Los Nodas.

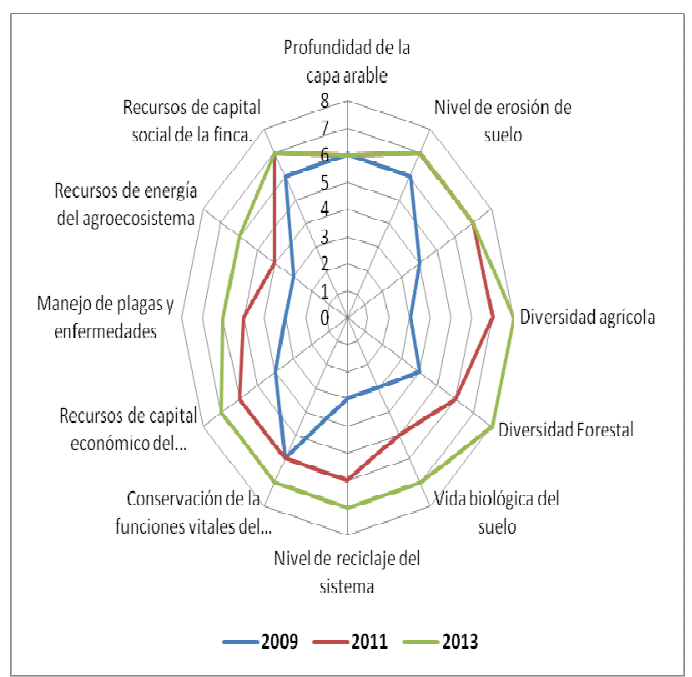

Finca Raúl Reyes

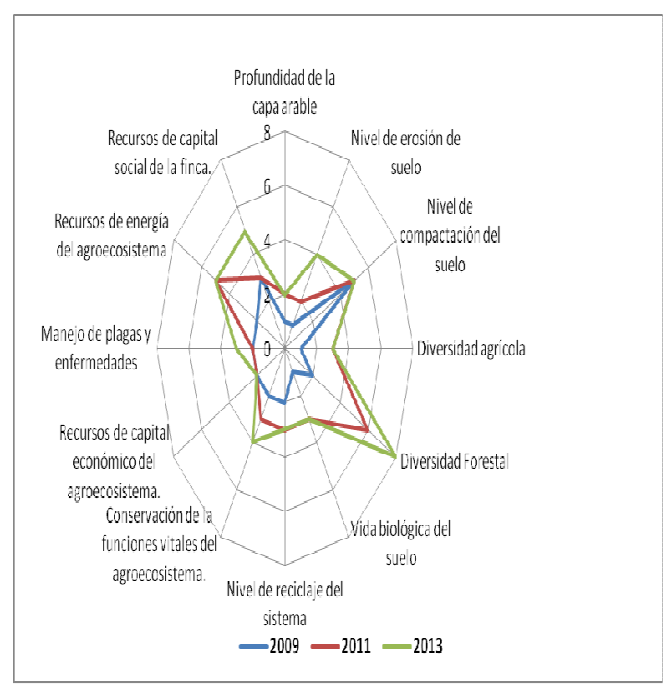

Finca Los Esperones 


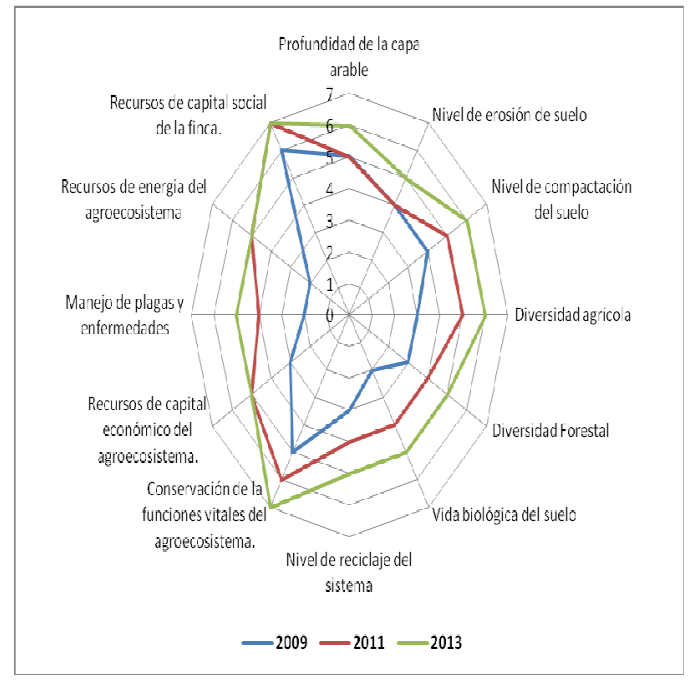

Finca Paco Hernández

El fortalecimiento de los sistemas agrícolas y su vinculación al manejo y gestión de los recursos naturales, ha logrado sensibilizar a las comunidades rurales sobre el enfoque agroecológicos de los sistemas tradicionales, disminuyendo la dependencia de estos de los recursos externos del sistema, logrando un mayor bienestar ambiental, econ \{omico y social de las familias rurales en la zona de amortiguamiento e influencia socioeconómica.

\section{CONSIDERACIONES FINALES}

El análisis de cada indicador dentro de los agroecosistemas estudiados, muestra que los avances de la propuesta agroecológica son paulatinos y parciales, existiendo mayores mejoras en las acciones y prácticas para la transformación del sistema tradicional al agroecológico, y en segundo plano, en las tecnologías de manejo para la conservación de los recursos naturales, ya que en el año 2013, todas las fincas contribuyeron a mejorar este indicador.

En el caso de las líneas relacionadas con la producción de alimentos para animales y la obtención de proteínas animales, estas aún son incipientes. Sin embargo, considerando, que en la totalidad de las fincas, se partía de un estado inicial en estos aspectos, debido a los sistemas agrícolas tradicionales que se desarrollaban en las mismas, se han conseguido logros importantes en la aplicación de las técnicas agroecológicas, por otro lado, se ha logrado también incorporar la participación de la mujer rural, no solo en el manejo de la finca, sino también, en la toma de decisiones. 
Fortalecimiento de los sistemas agrícolas productivos en la zona de amortiguamiento del Parque Nacional Viñales: una alternativa hacia el desarrollo sostenible de las comunidades rurales
Yoel Martínez Maqueira Ignacio H. Diaz-Marroto Ernesto Mujíca Benitez José Luis Corvea Porras Ana M. Castro Barrio

Antes de la aplicación de la metodología, las fincas no partían de la misma situación inicial, siendo, las que se encontraban en una peor situación, las siguientes: LEs (Los Esperones), EPa (El Paraíso) y PHz (Paco Hernández), mientras, que las fincas RRe (Raúl Reyes) y LEn (La Ensenada), mostraban la situación más favorable al inicio de la investigación.

En todas las fincas se produjo un incremento de los indicadores de sostenibilidad, mejorando sus agroecosistemas, excepto en la finca LEs (Los Esperones), que mantuvo un estado crítico en todo el proceso, solamente mejoró el indicador relacionado con la diversidad forestal.

\section{REFERENCIAS}

ALTIERI, M.A. Agroecology: the science of natural resource management for poor farmers in marginal environments. Agriculture, Ecosystems and Environment .2002, 93, 1-3: 1$24 p$.

ALTIERI, M.A. Linking ecologists and traditional farmers in the search for sustainable agriculture. Frontiers in Ecology and the Environment. 2004, v 2, (1): 35-42p.

ALTIERI, M.A., KOOHAFKAN, P. Enduring farms: climate change, smallholders and traditional farming communities. Environment and Development. 2008. Series 6. Third World Network, Penang, Malaysia.

ALTIERI, M.A., TOLEDO, V.M. The agroecological revolution in Latin America: rescuing nature, ensuring food sovereignty and empowering peasants. Journal of Peasant Studies. 2011. 38 (3): 587-612p.

CAPORAL, R., MORALES, J. Rio Grande do Sul,Vers l'agroecologie. L'Ecologiste. 2004. (3): 40-41p.

GLIESSMAN, S.R. Agroecology: Ecological Processes in Sustainable Agriculture.1998 Ann Arbor Press, Chelsea, MI.

GLIESSMAN, S.R. Agroecología: procesos ecológicos en la agricultura sostenible. CATIE: Turrialba, Costa Rica.2002.

GUZMAN, G. et al. Introducción a la agroecología como desarrollo rural sostenible. Ed. Mundi prensa, Madrid. 2000.

Holt-Gimenez, E. 2001. Measuring farms agroecological resistance to Hurricane Mitch. LEISA, 2001, 17: p18-20. 
Fortalecimiento de los sistemas agrícolas productivos en la zona de amortiguamiento del Parque Nacional Viñales: una alternativa hacia el desarrollo sostenible de las comunidades rurales
Yoel Martínez Maqueira Ignacio H. Diaz-Marroto Ernesto Mujíca Benitez José Luis Corvea Porras Ana M. Castro Barrio

HOLT-GIMENEZ, E., PATEL, R. Food rebellions: the real story of the world food crisis and what we can do about it. Fahumu Books and Grassroots International, Oxford, UK.2009.

KOOHAFKAN, P., ALTIERI, M.A. Globally Important Agricultural Heritage Systems: a legacy for the future. FAO, Rome, Italy.2011.

LEHMANN, H. et al. Karstmorphologische, geologische und botanische Studien in der Sierra De Los Organos auf Cuba. Erdkunde Archiv for Scientific Geographie 1956. 10 (3): 185$204 p$.

MACHIN et al. Revolución agroecológica: el movimiento campesino a campesino de la ANAP en Cuba. ANAP- Vía campesina, La Habana. 2010.

MARTINEZ, Y. 2013. Plan de manejo del Parque Nacional Viñales 2014-2020. Centro Nacional de Áreas Protegidas, La Habana, Cuba.

PRETTY et al. Reducing food poverty by increasing agricultural sustainability in the development countries, Agriculture. Ecosystems and Environment. 2003. 95 (1): 217$234 p$.

PRETTY, J.C. et al. Sustainable intensification in African Agriculture. International Journal of Agricultural Sustainability. 2011.9 (1): 5-24.

PSZCZOLKOWSKI, A. Secuencias miogeosinclinales de la Cordillera de Guaniguanico. Litoestratigrafía, desarrollo de facies y paleogeografía. En: PSZCZOLKOWSKI, A., PISTROWSKA, K., PIOTROWSKI, J., de la TORRE, A., MYCZYNSKI, R., HACZEWKI, G. (eds.). Contribución a la Geología de la Provincia de Pinar del Río.1987. La Habana. Editorial Científico Técnica, Cuba.

REILJ, C.P., SMALING, M. A. Analyzing successes in agriculture and land management in Sub-Saharan Africa: is macrolevel gloom obscuring positive microlevel change? 2008. Land Use Policy 25 (3): 410-420.

ROSSET, P.M. et al. The Campesino-to-Campesino agroecology movement of ANAP in Cuba: social process methodology in the construction of sustainable peasant agriculture and food sovereignty. Journal of Peasant Studies.2011. 38 (1): 161-91p.

SEVILLA-GUZMAN et al. Los marcos conceptuales de la agroecología. En: BEZERRA, M.A., TAVARES de Lima, J.R. (org.), Agroecología, Conceitos e Experiências. Ediciones Bagaço, Recife, Brasil.2006.

TOLEDO, V.M. The ecological rationality of peasant production. In: ALTIERI, M.A., HECHT, S. (eds.), Agroecology and small farmer development. CRC Press, Boca Ratón, Florida.1990. 
Fortalecimiento de los sistemas agrícolas productivos en la zona de amortiguamiento del Parque Nacional Viñales: una alternativa hacia el desarrollo sostenible de las comunidades rurales
Yoel Martínez Maqueira Ignacio H. Diaz-Marroto Ernesto Mujíca Benitez José Luis Corvea Porras Ana M. Castro Barrio

TOLEDO, V.M., BARRERA-BASSOLS, N. La Memoria biocultural: la importancia ecológica de las sabidurías tradicionales. ICARIA Editorial, Barcelona.2008.

UK FOOD GROUP. Securing future food: towards ecological food provision.2010.Agreed 3 july 2013 on line. Avalibale at: http://www.ukfg.org.uk/pdfs/Securing_future_food.pdf

UPHOFF, N. Agroecological innovations. Increasing food production with participatory development. Earthscan, London. 2002.

Recebido em 29/05/2017.

Aceito para publicação em 27/11/2017. 